\title{
ELIE CARTAN AND HIS MATHEMATICAL WORK
}

\author{
SHIING-SHEN CHERN AND CLAUDE CHEVALLEY
}

After a long illness Elie Cartan died on May 6, 1951, in Paris. His death came at a time when his reputation and the influence of his ideas were in full ascent. Undoubtedly one of the greatest mathematicians of this century, his career was characterized by a rare harmony of genius and modesty.

Elie Cartan was born on April 9, 1869 in Dolomieu (Isère), a village in the south of France. His father was a blacksmith. Cartan's elementary education was made possible by one of the state stipends for gifted children. In 1888 he entered the "Ecole Normale Supérieure," where he learned higher mathematics from such masters as Tannery, Picard, Darboux, and Hermite. His research work started with his famous thesis on continuous groups, a subject suggested to him by his fellow student Tresse, recently returned from studying with Sophus Lie in Leipzig. Cartan's first teaching position was at Montpellier, where he was "maitre de conférences"; he then went successively to Lyon, to Nancy, and finally in 1909 to Paris. He was made a professor at the Sorbonne in 1912. The report on his work which was the basis for this promotion was written by Poincaré; ${ }^{1}$ this was one of the circumstances in his career of which he seemed to have been genuinely proud. He remained at the Sorbonne until his retirement in 1940.

Cartan was an excellent teacher; his lectures were gratifying intellectual experiences, which left the student with a generally mistaken idea that he had grasped all there was on the subject. It is therefore the more surprising that for a long time his ideas did not exert the influence they so richly deserved to have on young mathematicians. This was perhaps partly due to Cartan's extreme modesty. Unlike Poincaré, he did not try to avoid having students work under his direction. However, he had too much of a sense of humor to organize around himself the kind of enthusiastic fanaticism which helps to form a mathematical school. On the other hand, the bulk of the mathematical research which was accomplished at the beginning of this century in France centered around the theory of analytic functions; this subject, made glamorous by the achievement represented

1 This report was in part published in Acta Math. vol. 38 (1921) pp. 137-145. It should be of considerable historic interest to have now a complete version of this report. 
by Picard's theorem, offered many not too difficult problems for a young mathematician to tackle. In the minds of inexperienced beginners in mathematics, Cartan's teaching, mostly on geometry, was sometimes very wrongly mistaken for a remnant of the earlier Darboux tradition of rather hollow geometric elegance. When, largely under the influence of $\mathrm{A}$. Weil, a breeze of fresh air from the outside came to blow on French mathematics, it was a great temptation to concentrate entirely on the then ultra-modern fields of topology or modern algebra, and the ideas of Cartan once more, though for other reasons, partially failed to attract the amount of attention which was their due. This regrettable situation was partly corrected when Cartan's work was taken (at the suggestion of A. Weil) in 1936 to be the central theme of the seminar of mathematics organized by Julia. In 1939, at the celebration of Cartan's scientific jubilee, J. Dieudonné could rightly say to him: " . . vous êtes un "jeune," et vous comprenez les jeunes"-it was then beginning to be true that the young understood Cartan.

In foreign countries, particularly in Germany, his recognition as a great mathematician came earlier. It was perhaps $H$. Weyl's fundamental papers on group representations published around 1925 that established Cartan's reputation among mathematicians not in his own field. Meanwhile, the development of abstract algebra naturally helped to attract attention to his work on Lie algebra. However, the reception of his contributions to differential geometry was varied. This was partly due to his approach which, though leading more to the heart of the problem, was unconventional, and partly due to inadequate exposition. Thus Weyl, in reviewing one of Cartan's books [41],2 wrote in 1938:" "Cartan is undoubtedly the greatest living master in differential geometry.... I must admit that I found the book, like most of Cartan's papers, hard reading. ..." This sentiment was shared by many geometers.

Cartan was elected to the French Academy in 1931. In his later years he received several other honors. Thus he was a foreign member of the National Academy of Sciences, U.S.A., and a foreign Fellow of the Royal Society. In 1936 he was awarded an honorary degree by Harvard University.

Closely interwoven with Cartan's life as a scientist and teacher has been his family life, which was filled with an atmosphere of happiness and serenity. He had four children, three sons, Henri, Jean,

${ }^{2}$ Numbers in brackets refer to the bibliography at the end of the paper.

3 Bull. Amer. Math. Soc. vol. 44 (1938) p. 601. 
and Louis, and a daughter, Hélène. Jean Cartan oriented himself towards music, and already appeared to be one of the most gifted composers of his generation when he was cruelly taken by death. Louis Cartan was a physicist; arrested by the Germans at the beginning of the Résistance, he was murdered by them after a long period of detention. There is no need to say here that Henri Cartan followed in the footsteps of his father to become a mathematician.

Cartan's mathematical work can be roughly classified under three main headings: group theory, systems of differential equations, and geometry. These themes are, however, constantly interwoven with each other in his work. Almost everything Cartan did is more or less connected with the theory of Lie groups.

S. Lie introduced the groups which were named after him as groups of transformations, i.e., as systems of analytic transformations on $n$ variables such that the product of any two transformations of the system still belongs to the system and each transformation of the system has an inverse in the system. The idea of considering the abstract group which underlies a given group of transformations came only later; it is more or less implicit in Killing's work and appears quite explicitly already in the first paper by Cartan. Whereas, for Lie, the problem of classification consisted in finding all possible transformation groups on a given number of variables - a far too difficult problem in the present stage of mathematics as soon as the number of variables is not very small-for Killing and Cartan, the problem was to find all possible abstract structures of continuous groups; and their combined efforts solved the problem completely for simple groups. Once the structures of all simple groups were known, it became possible to look for all possible realizations of any one of these structures by transformations of a specified nature, and, in particular, for their realizations as groups of linear transformations. This is the problem of the determination of the representations of a given group; it was solved completely by Cartan for simple groups. The solution led in particular to the discovery, as early as 1913, of the spinors, which were to be re-discovered later in a special case by the physicists.

Cartan also investigated the infinite Lie groups, i.e., the groups of transformations whose operations depend not on a finite number of continuous parameters, but on arbitrary functions. In that case, one does not have the notion of the abstract underlying group. Cartan and Vessiot found, at about the same time and independently of each other, a substitute for this notion of the abstract group which consists in defining when two infinite Lie groups are to be considered as 
isomorphic. Cartan then proceeded to classify all possible types of non-isomorphic infinite Lie groups.

Cartan paid also much attention to the study of topological properties of groups considered in the large. He showed how many of these topological problems may be reduced to purely algebraic questions; by so doing, he discovered the very remarkable fact that many properties of the group in the large may be read from the infinitesimal structure of the group, i.e., are already determined when some arbitrarily small piece of the group is given. His work along these lines resembles that of the paleontologist reconstructing the shape of a prehistoric animal from the peculiarities of some small bone.

The idea of studying the abstract structure of mathematical objects which hides itself beneath the analytical clothing under which they appear at first was also the mainspring of Cartan's theory of differential systems. He insisted on having a theory of differential equations which is invariant under arbitrary changes of variables. Only in this way can the theory uncover the specific properties of the objects one studies by means of the differential equations they satisfy, in contradistinction to what depends only on the particular representation of these objects by numbers or sets of numbers. In order to achieve such an invariant theory, Cartan made a systematic use of the notion of the exterior differential of a differential form, a notion which he helped to create and which has just the required property of being invariant with respect to any change of variables.

Raised in the French geometrical tradition, Cartan had a constant interest in differential geometry. He had the unusual combination of a vast knowledge of Lie groups, a theory of differential systems whose invariant character was particularly suited for geometrical investigations, and, most important of all, a remarkable geometrical intuition. As a result, he was able to see the geometrical content of very complicated calculations, and even to substitute geometrical arguments for some of the computations. The latter practice has often been baffling to his readers. But it is an art whose presence is usually identical with the vigor of a geometrical thinker.

In the 1920's the general theory of relativity gave a new impulse to differential geometry. This gave rise to a feverish search of spaces with a suitable local structure. The most notable example of such a local structure is a Riemann metric. It can be generalized in various ways, by modifying the form of the integral which defines the arc length in Riemannian geometry (Finsler geometry), by studying only those properties pertaining to the geodesics or paths (geometry 
of paths of Eisenhart, Veblen, and T. Y. Thomas), by studying the properties of a family of Riemann metrics whose fundamental forms differ from each other by a common factor (conformal geometry), etc. While in all these directions the definition of a parallel displacement is considered to be the major concern, the approach of Cartan to these problems is most original and satisfactory. Again the notion of group plays the central rôle. Roughly speaking, a generalized space (espace généralisé) in the sense of Cartan is a space of tangent spaces such that two infinitely near tangent spaces are related by an infinitesimal transformation of a given Lie group. Such a structure is known as a connection. The tangent spaces may not be the spaces of tangent vectors. This generality, which is absolutely necessary, gave rise to misinterpretation among differential geometers. As we shall show below, it is now possible to express these concepts in a more satisfactory way, by making use of the modern notion of fiber bundles.

We can perhaps conclude from the above brief description that Cartan's mathematical work, unlike that of Poincaré or Hadamard, centers around a few major concepts. This is partly due to the richness of the field, in which his pioneering work has opened avenues where much further development is undoubtedly possible. While many of Cartan's ideas have received clarification in recent years, the difficulties of conceiving the proper concepts at the early stage of development can hardly be overestimated. Thus in writing on the psychology of mathematical thinking, Hadamard had to admit "the insuperable difficulty in mastering more than a rather elementary and superficial knowledge of Lie groups." Thanks to the development of modern mathematics, such difficulties are now eased.

Besides several books Cartan published about 200 mathematical papers. It is earnestly to be hoped that the publication of his collected works may be initiated in the near future. Not only do they fully deserve to find their place on the bookshelves of our libraries at the side of those of other great mathematicians of the past, but they will be, for a long time to come, a most indispensable tool for all those who will attempt to proceed further in the same directions.

We now proceed to give a more detailed review of some of the most important of Cartan's mathematical contributions.

\section{GROUP THEORY}

Cartan's papers on group theory fall into two categories, distinguished from each other both by the nature of the questions

' J. Hadamard, The psychology of invention in the mathematical field, Princeton, 1945, p. 115. 
treated and by the time at which they were written. The papers of the first cycle are purely algebraic in character; they are more concerned with what are now called Lie algebras than with group theory proper. In his thesis [3], Cartan gives the complete classification of all simple Lie algebras over the field of complex numbers. They fall into four general classes (which are the Lie algebras of the unimodular groups, of the orthogonal groups in even or odd numbers of variables, and of the symplectic groups) and a system of five "exceptional" algebras, of dimensions 14, 52, 78, 133, and 248. Killing had already discovered the fact that, outside the four general classes, there can exist only these five exceptional Lie algebras; but his proofs were incorrect at several important points, and, as to the exceptional algebras, it is not clear from his paper whether he ever proved that they actually existed. Moreover, in his work, the algebra of dimension 52 appears under two different forms, whose equivalence he did not recognize. Cartan gave rigorous proofs that the classification into four general classes and five exceptional algebras is complete, and constructed explicitly the exceptional algebras.

Let $\mathfrak{g}$ be any Lie algebra; to every element $X$ of $\mathfrak{g}$ there is associated a linear transformation, the adjoint ad $X$ of $X$, operating on the space $\mathfrak{g}$, which transforms any element $Y$ of $\mathfrak{g}$ into $[X, Y]$. Because of the relation $[X, X]=0$, this linear transformation always admits 0 as a characteristic root; those elements $X$ of $\mathfrak{g}$ for which 0 is a characteristic root of least possible multiplicity of ad $X$ are called regular elements. Let $H$ be a regular element; then those elements of $\mathfrak{g}$ which are mapped into 0 by powers of ad $H$ are seen to form a certain subalgebra $\mathfrak{h}$ of $\mathfrak{g}$, and this subalgebra is always nilpotent (which means that, in the adjoint representation of such an algebra, every element has 0 as its only characteristic roots). A subalgebra such as $\mathfrak{h}$ has been called a Cartan subalgebra of $\mathfrak{g}$. It is a kind of inner core of the algebra $\mathfrak{g}$, and many properties of the big algebra $\mathfrak{g}$ are reflected in properties of this subalgebra $\mathfrak{h}$. In the case where $\mathfrak{g}$ is semi-simple, $\mathfrak{h}$ is always abelian (which means, for a Lie algebra, that $[X, Y]$ is always 0 for any $X$ and $Y$ in the algebra). Moreover, $\mathfrak{g}$ has a base which is composed of elements which are eigenvectors simultaneously for all adjoint operations of elements of $\mathfrak{h}$. The factors by which these elements are multiplied when bracketed with elements of $\mathfrak{h}$ are called the roots of the Lie algebra; it is the study of the properties of these roots which leads to the classification of simple Lie algebras. In establishing these properties, Cartan made a systematic use of the "fundamental quadratic form" of $\mathfrak{g}$, whose value at an element $X$ is the trace of the square of ad $X$ (if $g$ is 
semi-simple, or more generally if it coincides with its derived algebra, then the trace of ad $X$ itself is always zero). One of the most important results of Cartan's thesis is that a necessary and sufficient condition for $\mathfrak{g}$ to be semi-simple is that its fundamental quadratic form be nondegenerate (i.e. that its rank be equal to the dimension of g). Incidentally, Cartan also applied similar methods to the study of systems of hypercomplex numbers (cf. [4]) and obtained in this manner the main structure theorems for associative algebras over the fields of real and of complex numbers; however, these results were superseded by the work of Wedderburn, which applies to algebras over arbitrary basic fields. By studying those algebras which have only one integrable (or, as we say now, solvable) ideal, Cartan also laid the foundations in his thesis for his subsequent study of linear representations of simple Lie algebras; in particular, he determined, for each class of simple groups, the linear representation of smallest possible degree.

The general theory of linear representations is the object of the paper [5]. As above let $g$ be any semi-simple Lie algebra over the field of complex numbers (any algebraically closed field of characteristic 0 would do just as well); a linear representation of $\mathfrak{g}$ is a law which assigns to every $X$ in $\mathfrak{g}$ a linear transformation $\rho(X)$ on some finitedimensional space; $\rho(X)$ depends linearly on $X$, and is such that $\rho([X, Y])=\rho(X) \rho(Y)-\rho(Y) \rho(X)$ for any $X$ and $Y$ in $\mathfrak{g}$. Let $\mathfrak{h}$ be a Cartan subalgebra of $g$. Then it turns out that the matrices which represent the elements of $\mathfrak{h}$ may all be reduced simultaneously to the diagonal form; the diagonal coefficients which occur in these matrices, considered as linear functions of the element which is represented, are called the weights of the representation. The roots of $g$ are the weights of a particular linear representation, viz. the adjoint representation. Cartan proved that all relations between weights of one or several representations are consequences of certain linear relations with rational coefficients between these weights, a fact which can now be explained in two different manners: it reflects the properties of characters of compact abelian groups, and also the properties of algebraic groups of linear transformations. Cartan then introduces an order relation in the system of all weights and roots, and proves that any irreducible representation is uniquely determined by its highest weight for this order relation. The problem of finding all irreducible linear representations of $\mathfrak{g}$ is thereby reduced to that of finding all possible highest weights of representations. The sum of the highest weights of two irreducible representations is again the highest weight of an irreducible representation, which is contained in the tensor 
product (or rather, sum, if we speak of representations of Lie algebras and not of groups) of the two given representations. If $r$ is the rank of the Lie algebra $g$ (i.e. the dimension of any Cartan subalgebra of g), Cartan established that all possible highest weights of irreducible representations may be written as linear combinations with nonnegative integral coefficients of $r$ particular linear functions which depend only on the structure of $\mathfrak{g}$ and the order relation in the system of roots. Considering one by one the various types of simple Lie algebras, he established that every one of these $r$ basic functions is the highest weight of some irreducible representation; this led to a complete classification of all irreducible linear representations of simple Lie algebras. This theory of linear representations was later completed in an important point by $\mathrm{H}$. Weyl, who established by transcendental methods that every representation of a semi-simple Lie algebra is completely reducible, and who expressed the degree of an irreducible representation in terms of its highest weight. It has also recently become possible to give a direct proof of the existence of irreducible representations corresponding to the possible highest weights predicted by Cartan's theory, a proof which applies not only to simple but also to semi-simple algebras (Lie algebras behave differently from associative algebras in this respect that algebras which are not simple may have faithful irreducible representations; this happens for instance for the Lie algebra of the orthogonal group in 4 variables). In the process of classifying all possible linear representations, Cartan discovered the spin representations of the orthogonal Lie algebras, which later played such an important rôle in physics. In a book published later (Leçons sur la thêorie des spineurs, Hermann, Paris, 1938), Cartan developed the theory of spinors from a geometric point of view.

In [6] Cartan classifies all simple Lie algebras over the field of real numbers instead of that of complex numbers. The method is to study the "complexification" of the Lie algebra under consideration; this complexification is either simple or the sum of two simple Lie algebras, and there is defined in it an operation of passing to the imaginary conjugate which admits the elements of the given simple Lie algebra as fixed elements. Starting with a suitable complex algebra, Cartan determines all possible operations of conjugation in it and arrives in this manner at a complete classification of simple real Lie algebras (the method was later simplified by himself and by others, making use of the compact real forms and replacing the determination of all possible conjugations by that of all classes of involutive automorphisms of the compact form). 
It turned out that, but for one exception, the structure of a simple real Lie algebra is characterized by that of its complexification and by its character, i.e., by the index of inertia of its fundamental quadratic form. Cartan noticed that, for every complex form, there is a unique real form whose fundamental quadratic form is negative definite; this real form is the Lie algebra of a compact Lie group. This fact was to play a very important rôle in the subsequent theory of Lie groups, because it establishes a one-to-one correspondence between semi-simple connected complex Lie groups and compact semi-simple Lie groups. While the former are more readily amenable to an algebraic study, because of the algebraically closed character of the basic field, the latter lend themselves more easily to study by transcendental methods because the volume of the whole group (in the sense of Haar measure) is finite.

The last paper of the first cycle is [7] in which Cartan determined all real linear representations of simple real Lie algebras.

The work of Cartan's second group-theoretic period is concerned with the groups themselves, and not with their Lie algebras, and in general with the global aspect of the group. This period opens with a paper [8] which contains, a study of group manifolds from a local differential geometric point of view. A group $G$ may operate on itself in three different ways: by the left translations, by the right translations, and by transformation (we call here transformation a mapping $t \rightarrow s t s^{-1}$, where $s$ is a fixed element of the group). In relation to this, Cartan shows that there are three affine connections which are intrinsically defined on $G$. Two of these connections (those which correspond to the left and right translations) are without curvature but in general have torsion; the third one has no torsion but has in general a curvature. The geodesic lines are the same for all three connections: they are the cosets with respect to the one-parameter subgroups. Cartan determines also the totally geodesic varieties on $G$ (varieties such that any geodesic which is tangent to it is entirely contained in it); they are of two different kinds. The varieties of the first kind are the subgroups of $G$ and their cosets. The varieties of the second kind are determined by what have since been called the Lie triple systems contained in the Lie algebra $g$ of $G$, i.e., the linear subspaces of $g$ which, together with three elements $X, Y$, and $Z$, contain the element $[[X, Y], Z]$.

After the paper we have just mentioned, Cartan's interest orients itself very definitely towards the topological study of Lie groups in the large.

This period begins around 1925, at the time when $\mathrm{H}$. Weyl had 
just published his fundamental papers on the theory of compact Lie groups. It is difficult to appreciate to what extent Cartan was influenced by Weyl's methods and results; at any rate his book Leçons sur la Géométrie des espaces de Riemann shows clearly that, even before Weyl's paper, Cartan was already getting more and more interested in topological questions. Whereas Weyl's line of attack was, if we may say so, brutally global, depending essentially on the method of integration on the whole group, the work of Cartan puts the emphasis on the connection between the local and the global. This essential difference has a great bearing on the nature of the results it is possible to expect from these two methods. Weyl's methods are not bound to the differentiable structure of the group under consideration; as soon as the possibility of integrating on any locally compact group was established Weyl's results could be extended to all compact topological groups. However, the assumption of compactness is essential (it insures the convergence of the integrals on the group), and Weyl's methods give nothing on noncompact groups, whereas those of Cartan, applied in the domain of Lie groups, have led to a very complete knowledge of the topology of these groups, whether compact or not.

In his paper [9] Cartan studies the topology of compact semisimple Lie groups and of their complexifications. Let $G$ be a compact semi-simple Lie group, $\mathfrak{g}$ the Lie algebra of $G$, and $\mathfrak{h}$ a Cartan subalgebra of $\mathfrak{g}$. Cartan establishes that every element of $G$ belongs to a one-parameter subgroup (at least), and that every infinitesimal transformation may be transformed by an operation of the adjoint group into an element of $\mathfrak{h}$. Every element $H$ of $\mathfrak{h}$ gives rise to a oneparameter subgroup with a definite parameter on it; let us denote by $\exp H$ the point of parameter 1 on this subgroup. Then every element of $G$ is conjugate to some element of the form $\exp H$; the next question is to find out under which condition two elements exp $H$ and $\exp H^{\prime}$ of this form are conjugate to each other. Cartan shows that a necessary and sufficient condition for this to happen is that $H^{\prime}$ can be transformed into $H$ by an operation of a certain discontinuous group $S$ operating on the space $\mathfrak{h}$. This group admits a fundamental domain which is a polyhedron $P$ with a finite number of vertices. The points of $P$, with suitable identification of faces, will represent in a one-to-one manner the classes of conjugate elements of $G$. The inner points of $P$ correspond to the regular elements of the group, i.e., to those elements which may be represented in the form $\exp H$, where $H$ is a regular element of $\mathfrak{h}$. It follows that any closed path in $G$ which does not meet the set of singular (i.e., not regular) 
elements will be represented by a continuous path in $P$, not necessarily closed. Now, it turns out that the singular elements of $G$ form a set of dimension at least 3 less than the dimension of the whole group; as a consequence they may be entirely disregarded in the determination of the fundamental group. This allows one to proceed to the determination of this fundamental group $\pi$ by the mere consideration of the polyhedron $P$ itself; the order of $\pi$ is the number of vertices $H$ of $P$ such that $\exp H$ is the unit element of $G$. From this follows Weyl's theorem to the effect that the fundamental group of a compact semi-simple Lie group is finite. This implies that, if the fundamental quadratic form of a Lie group $G$ is definite negative, then not only does there exists at least one compact group which is locally isomorphic with $G$, but $G$ itself is compact. Moreover Cartan's methods allow one to determine, for every semi-simple infinitesimal structure with a negative definite fundamental quadratic form, the number of times that the simply-connected group with this structure covers the adjoint group, and to study the various types of closed one-parameter groups (or geodesics) in the space of a compact group. The last part of the paper, devoted to the study of simple complex groups, is a prelude to the future theory of noncompact simple groups; it is proved in particular that, for any simple Lie algebra over the complex numbers, there always exists a simplyconnected complex linear group having this Lie algebra.

After the determination of the fundamental group was accomplished, the next step was to determine the higher-dimensional Betti numbers of compact Lie groups. The method of accomplishing this was indicated in Cartan's paper [11]. Cartan considers a homogeneous space $E$ whose group of transformations $G$ is compact; the space may then be considered to be the space of cosets of $G$ modulo some closed subgroup $g$. Let us say that two exact differential forms are equivalent when their difference is the differential of a form of degree $p-1$, and let $b_{p}$ be the maximal number of forms of degree $p$ no linear combination of which is equivalent to 0 . It was conjectured, but not yet proved at the time, that $b_{p}$ is equal to the $p$ th Betti number of the space $E$ (this was established soon afterwards by de Rham). Let $\omega$ be any exact form of degree $p$. Any operation $s$ of the group $G$ transforms into a new exact form $s \omega$, which Cartan proves to be equivalent to $\omega$. He then constructs the average of the form $s \omega, s$ running over all elements of the group $G$; this new form is still exact, is equivalent to $\omega$, and is furthermore invariant under the group $G$. Moreover, Cartan proves by a similar argument that, if $\omega$ is invariant and equivalent to 0 , then $\omega$ is the differential of a form which is it- 
self invariant. These theorems reduce the determination of the number $b_{p}$ to that of the integral invariants of the space $E$. Cartan then shows that the latter problem may be reduced to a purely algebraic problem depending only on the Lie algebra of the group $G$ and the subalgebra corresponding to the subgroup $g$ (this, at any rate, in the case where the group $g$ is connected). This algebraic problem has been solved since for the case where $E$ is either the group $G$ itself or a symmetric Riemann space with $G$ as its group of isometric transformations.

We now come to the results obtained by Cartan in the study of Lie groups which are not compact. The proof he gave of the converse of Lie's third theorem (i.e., every Lie algebra over the field of real numbers is the Lie algebra of some group) implied that every simplyconnected Lie group is topologically the product of a Euclidean space by the space of a simply connected semi-simple group. The problem was therefore reduced to the special case in which the group $G$ under consideration is semi-simple. Let then $\mathfrak{g}$ be its Lie algebra, and let $\mathfrak{g}^{\prime}$ be the complexification of $\mathfrak{g}$. Since $\mathfrak{g}^{\prime}$ is semi-simple, it has a compact real form $\mathfrak{g}_{c}$; i.e., it may be considered as the complexification of a Lie algebra $g_{c}$ which is the algebra of a compact semi-simple group. Cartan proves (in his paper [10]) that there exists an involutory automorphism $a$ of $g_{c}$ such that $\mathfrak{g}$ is spanned by those elements of $\mathfrak{g}_{c}$ which are invariant under $a$ and by the products by $i$ of those elements of $g_{c}$ which are changed into their opposites by $a$. The subalgebra $\mathfrak{g} \cap \mathfrak{g}_{c}$ is the Lie algebra of a maximal compact subgroup $g$ of $G$. Cartan then considers the space $R$ whose points are the conjugates of $g$ in $G$; the essential fact is that the existence of the automorphism $a$ implies that $R$ is a symmetric Riemannian space, having the adjoint group $\Gamma$ of $G$ as its group of isometries (more precisely, $\Gamma$ is the component of the identity in the group of isometries of $R$ ). This opened the way to the application of the theory of symmetric Riemannian spaces which Cartan had already developed for its own merits (cf. the part of this article which is concerned with the geometric aspects of Cartan's work). Assume that $G$ is $\Gamma$ itself. Then every operation of $G$ may be decomposed uniquely into a rotation around the origin (the origin in $R$ being the point which represents the group $g$ itself) and a transvection. The transvections are the operations of $G$ which result from the integration of those infinitesimal transformations which are changed into their negatives by the operation $a$. They form a subvariety of $G$, homeomorphic to $R$, and it turns out that this variety has the topological structure of a Euclidean space. This establishes that the adjoint group of any con- 
nected semi-simple Lie group is topologically the product of the space of a compact group by a Euclidean space. Moreover, making use of the fact that any compact group of isometric transformations of the space $R$ admits a fixed point, Cartan proves that every compact subgroup of $G$ is conjugate to a subgroup of $g$. These results relative to the adjoint groups may be extended without any difficulty to the simply-connected groups with the same infinitesimal structure; they are also true for all intermediary groups, as follows from the recent work of Iwasawa.

\section{Systems OF DIFFERENTIAL EQUATIONS}

The principal paper of Cartan on the theory of differential systems is [15]. The reader will find a very clear exposition of the theory of Pfaffian systems in Cartan's book: Les systèmes différentiels extérieurs et leurs applications gêométriques, Paris, Hermann, 1945.

A Pfaffian system is a system consisting of a certain number of equations of the form $A_{1} d x_{1}+\cdots+A_{n} d x_{n}=0$, where $A_{1}, \cdots, A_{n}$ are functions of $x_{1}, \cdots, x_{n}$, and possibly of certain equations of the form $F\left(x_{1}, \cdots, x_{n}\right)=0$. A parametric $r$-dimensional manifold, given by $x_{i}=f_{i}\left(t_{1}, \cdots, t_{r}\right)(1 \leqq i \leqq n)$ is a solution of the system if the equations of the system become identically satisfied when one replaces the variables $x$ and their differentials by their expressions in terms of the variables $t$ and their differentials.

It is first necessary to indicate how any system of differential equations may be reduced to a Pfaffian system. If the system contains equations of order higher than one, we may first reduce it to the order one by introducing new unknown functions which represent certain derivatives of the original ones. This being done, we obtain a system of equations of the form $F_{i}\left(x_{1}, \cdots, x_{m} ; z_{1}, \cdots, z_{p} ; \cdots\right.$, $\left.\partial z_{r} / \partial x_{s}, \cdots\right)=0$ where the $z$ 's are the unknown and the $x$ 's the independent variables. If we set $\partial z_{r} / \partial x_{s}=t_{r s^{\prime}}$, the original system of partial differential equations may be replaced by the Pfaffian system composed of the equations $F_{i}(x ; z ; t)=0, d z_{r}-\sum_{s} t_{r s} d x_{s}=0$. The solutions of the original system correspond to those solutions of the Pfaffian system which are manifolds of dimension $r$ on which the variables $x_{1}, \cdots, x_{n}$ are independent.

The essential originality of Cartan consists in having introduced, besides the Pfaffian forms, the exterior differential forms of higher degree. The algebra of exterior forms had been developed by Grassmann for geometric purposes; before it could be used in the theory of differential systems, it was necessary to introduce the operation of exterior differentiation. An exterior differential form is an expres- 
sion of the form $\sum A_{i_{1} \cdots i_{p}} d x_{i_{1}} \cdots d x_{i_{p}}$, where the coefficients $A_{i_{1} \cdots i_{p}}$ are functions of the variables $x$; such expressions may be multiplied with each other with the convention that $d x_{i} d x_{j}=-d x_{j} d x_{i}$ (in particular, $\left.\left(d x_{i}\right)^{2}=0\right)$. The exterior differential of the form written above is $\sum d A_{i_{1} \cdots i_{p}} d x_{i_{1}} \cdots d x_{i_{p}}$ where it is understood that the differentials $d A_{i_{1} \cdots i_{p}}$ are expressed as linear combinations of $d x_{1}, \cdots, d x_{n}$. The fundamental property of the operation of differentiation is that it is invariant with respect to any change of variables. Now, let us consider any Pfaffian system $\omega_{1}=0, \cdots, \omega_{h}=0$, $F_{1}=0, \cdots, F_{m}=0$, where $F_{1}, \cdots, F_{m}$ are functions and $\omega_{1}, \cdots, \omega_{h}$ Pfaffian forms; then we see immediately that any solution of the system will also be a solution of the system obtained by adjoining to the original one the equations $d F_{1}=0, \cdots, d F_{m}=0, d \omega_{1}=0, \cdots$, $d \omega_{h}=0$. More generally, let $I$ be the smallest set of differential forms containing $F_{1}, \cdots, F_{m}, \omega_{1}, \cdots, \omega_{h}$ and such that, whenever $\omega$ and $\omega^{\prime}$ are in $I$, then $\omega+\omega^{\prime}$ is in $I$, the product of $\omega$ by an arbitrary differential form is in $I$, and $d \omega$ is in $I ; I$ is called the differential ideal generated by $F_{1}, \cdots, F_{m}, \omega_{1}, \cdots, \omega_{h}$. Then any solution of the original system will be a solution of the system obtained by equating to 0 all forms in $I$. The operation of adjoining to a system the exterior differentials of its forms is an invariant counterpart of the method of obtaining conditions of compatibility by writing that certain higher derivatives which may be computed in two different ways by means of the equations of the system have the same value.

By a contact element $E_{p}$ of dimension $p$ in the Cartesian $n$-space $R^{n}$ is meant a pair $(M, P)$ formed by a point $M$ of $R^{n}$ and a $p$-dimensional linear subspace $P$ of $R^{n}$ going through $M$. It is sometimes necessary to generalize this notion to the case of an arbitrary $n$-dimensional manifold $V$ instead of $R^{n} ; M$ is then any point of $V$, and $P$ any $p$-dimensional vector subspace of the $n$-dimensional tangent vector space to $V$ at $M$. The totality of all $p$-dimensional contact elements of a given manifold $V$ is itself a manifold $V_{1}$, the so-called first prolonged manifold of $V$. If $x_{1}, \cdots, x_{n}$ are local coordinates at the origin of the contact element $(M, P), P$ may be represented parametrically by equations of the form $d x_{i}=L_{i}\left(v_{1}, \cdots, v_{p}\right)$, where the $L_{i}$ 's are linear forms in $p$ parameters $v_{1}, \cdots, v_{p}$. Let $\omega$ be a differential form; if we substitute the coordinates of the origin of a contact element $E_{p}$ for the variables $x_{1}, \cdots, x_{n}$ in the coefficients of $\omega$, and the linear forms $L_{i}$ for the differentials $d x_{i}$ which occur in $\omega$, we obtain an exterior form in the variables $v$. If this form is 0 , then we say that $\omega$ is 0 at $E_{p}$. If we are given a differential ideal $I$ of dif- 
ferential forms, and if every form of $I$ is 0 at $E_{p}$, then we say that $E_{p}$ is an integral element of $I$.

Let $W$ be a $p$-dimensional submanifold of $V$. If $M$ is a point of $W$, we may represent $W$ locally around $M$ by equations of the form $x_{i}=f_{i}\left(u_{1}, \cdots, u_{p}\right)$, the $u_{i}$ 's being parameters. The contact element $(M, P)$ formed by $M$ and by the $p$-dimensional tangent space $P$ to $W$ at $N$ is called the tangential element of $W$ at $M$; the space $P$ may be represented by the equations $d x_{i}=d f_{i}$, the $d u_{i}$ 's taking the place of the parameters $v_{i}$ considered above. A solution (or integral manifold) of the system obtained by equating to 0 the forms of a differential ideal $I$ is a manifold $W$ whose tangential contact elements are integral elements of $I$. The problem of looking for such solutions may be decomposed into two parts: the determination of all integral elements, which is an algebraic problem, and the determination of the ways of grouping these integral elements together in such a way that they may be the tangential elements of some manifold.

The ideal $I$ may contain forms of degree 0 , i.e., functions of the variables. Assume that these functions, equated to 0 , represent an irreducible analytic manifold $V_{0}$. Assume that any point of $V_{0}$ is the origin of $\infty \boldsymbol{r}_{1}$ integral elements of dimension 1 . There may be points of $V$ which are the origins of more than $\infty r_{1}$ integral elements of dimension 1, but they form lower-dimensional submanifolds of $V$. A point of $V$ which does not lie on any one of these submanifolds is called an ordinary point. The smallest manifold (in the space of integral elements of dimension 1) which contains the integral elements whose origins are ordinary points is called the manifold of general integral elements of dimension 1; the origin of a general integral element is not necessarily ordinary. Assume now that any general integral element of dimension 1 is contained in $\infty$ integral elements of dimension 2; then those integral elements of dimension 1 which are not contained in more than $\infty^{r_{2}}$ integral elements of dimension 2 are called ordinary; proceeding as above, we define the notion of a general integral element of dimension 2 . We may continue in the same manner, and define inductively the integers $r_{1}, r_{2}, \cdots, r_{n}$. For a certain dimension $n$, the $r_{n+1}$ will be 0 , which means that not every general integral element of dimension $n$ will be contained in an integral element of dimension $n+1$. The number $n$ is called the genus of the system, and the system is said to be in involution for every dimension not greater than $n$. A p-dimensional general integral element $E_{p}$ is called regular if there exists a chain $E_{0} \subset E_{1} \subset \cdots$ 
$C E_{p-1} \subset E_{p}$ where, for each $i<p, E_{i}$ is an ordinary general integral element of dimension $i$. A $p$-dimensional general solution of $I$ is a $p$-dimensional manifold whose tangential contact elements are general integral elements of $I$, at least one of these integral elements being regular. In this manner, Cartan succeeded for the first time in giving a precise definition of the notion of the general solution of any differential system. The existence theorem for general solutions states that any regular $p$-dimensional integral element $E_{p}$ is a tangential contact element of some manifold which is a solution of $I$. More precisely, if $E_{p-1}$ is a regular element of dimension $p-1$ contained in $E_{p}$ and is a tangential element of a $(p-1)$-dimensional integral manifold $V^{p-1}$ of $I$, then $V^{p-1}$ is contained in at least one integral manifold $V^{p}$ tangent to $E_{p}$. This general theorem allowed Cartan to determine exactly the degree of indetermination of the general solution (i.e. on how many arbitrary constants, arbitrary functions of $1,2, \cdots$ arguments, it depends). Its application is however limited to the consideration of analytic differential systems and to the determination of analytic solutions.

The next step was to try to determine the singular solutions of the system, i.e. the solutions which are not in the general solution (for instance, for a differential equation in the plane, the envelope of the general solutions). Here the idea of Cartan was to construct from the given differential system new systems which are obtained from it by a method of prolongation, in such a manner that any singular solution of the original system should become a general solution of one of these new systems. The method consists, generally speaking, in adjoining new unknowns which are the coordinates of integral elements which are not general, and constructing $a$ priori the finite and differential equations these new unknowns must satisfy. However, an exact description of the method would be a little too long to be given here. In every concrete case in which it was applied, Cartan's method led to the complete determination of all singular solutions. But a general proof that it always does so is still missing; this is a theme of research which would richly deserve to attract the attention of ambitious young mathematicians.

One of the main applications of Cartan's theory of systems of differential equations is his theory of infinite groups of transformations (cf. $[16 ; 17 ; 18 ; 19])$. We touch here a branch of mathematics which is very rich in results but which very badly needs clarification of its foundations. For the infinite Lie groups, in spite of their name, are probably no groups at all, in the precise sense the word has received in modern algebra; what they really are is not clear yet. Lie defined 
them as follows: he considers a set of analytic transformations $x_{i}^{\prime}=F_{i}\left(x_{1}, \cdots, x_{n}\right)$ on $n$ variables which is closed with respect to the ordinary operations of forming the product of two transformations and taking the inverse of a transformation of the set, and which has furthermore the property of being composed of all transformations of the form indicated above for which the functions $F_{1}$ satisfy a certain system of partial differential equations. The hitch is of course that nothing is said about the domains in which the transformations are to be defined and invertible, and that this domain may apparently vary from one transformation to another. Cartan establishes that any Lie group, whether finite or infinite, may be defined (after possible adjunction of new variables, which transform in a suitable way when the original variables are transformed by an operation of the group) to be the group of all transformations which leave invariant a certain number of functions and Pfaffian forms. A simple but not typical example is the group of transformations of the form $x^{\prime}=F(x), y^{\prime}=G(y)$ on two variables $x$ and $y$ (where $F$ and $G$ are arbitrary analytic functions). It may be considered to be the group which leaves invariant the two Pfaffian forms $u d x$ and $v d y$, where $u$ and $v$ are new variables, which are transformed as follows: we have $u^{\prime}=u(d F / d x)^{-1}, v^{\prime}=v(d G / d x)^{-1}$. Having written a group in the form we have just indicated, Cartan was able to extend to infinite groups the structure theory which Lie had developed for finite groups. Assume that we have a group $G$ which is defined by the conditions that some of the variables, say $x_{r+1}, \cdots, x_{n}$, are invariant and that some Pfaffian forms $\omega_{1}, \cdots, \omega_{h}$ are invariant, the $\omega_{i}$ 's containing only the differentials of the variables $x_{1}, \cdots, x_{n}$ but their coefficients involving possibly certain other variables $u$. Then we may write

$$
d \omega_{i}=\sum c_{i j k} \omega_{j} \omega_{k}+\sum a_{i j k} \omega_{j} \eta_{k}
$$

where the $\eta_{k}$ 's are certain linear combinations of the differentials of the auxiliary variables $u$. Cartan shows that it is always possible to assume that the coefficients $c_{i j k}, a_{i j k}$ depend only on the invariants $x_{r+1}, \cdots, x_{n}$ (if the group may be put into a form where it is transitive, which always happens for finite-dimensional groups, then the $c_{i j k}$ 's and $a_{i j k}$ 's are constants). These coefficients define the structure of the group. Just as in the case of finite-dimensional groups, they cannot be taken arbitrarily; Cartan gave the conditions they must satisfy in order to define a group, thus generalizing to infinite groups Lie's third fundamental theorem.

The operation of adjoining new variables to those which are transformed by a group is called the prolongation of the group. Cartan 
says that two groups are isomorphic to each other if they admit prolongations which are similar, i.e., which can be deduced from each other by a change of independent variables. He showed how it is possible to recognize whether two infinite groups whose structures are known are isomorphic or not. He applied this method to the problem of the classification of simple infinite groups, and found that they fall into 8 general types.

Cartan's theory of infinite groups had its origin in the study he made of equivalence problems. The general problem can be formulated as follows: Let $G$ be a linear group acting in a space of $n$ dimensions. Let $\theta_{1}, \cdots, \theta_{n}$ and $\bar{\theta}_{1}, \cdots, \bar{\theta}_{n}$ be two sets of linearly independent Pfaffian forms in the variables $x_{1}, \cdots, x_{n}$ and $\bar{x}_{1}, \cdots$, $\bar{x}_{n}$, respectively. Determine whether there exists an admissible transformation of coordinates

$$
\bar{x}_{i}=\bar{x}_{i}\left(x_{1}, \cdots, x_{n}\right), \quad i=1, \cdots, n,
$$

such that

$$
\bar{\theta}_{i}=\sum_{j=1}^{n} a_{i j}(x) \theta_{j}, \quad i=1, \cdots, n,
$$

where the linear transformation belongs to $G$. To treat this problem let $u_{1}, \cdots, u_{m}$ be the parameters of $G$ and let us introduce the Pfaffian forms

$$
\begin{aligned}
& \omega_{i}=\sum_{j=1}^{n} a_{i j}(u) \theta_{j}, \\
& \bar{\omega}_{i}=\sum_{j=1}^{n} a_{i j}(\bar{u}) \bar{\theta}_{j}, \quad i=1, \cdots, n,
\end{aligned}
$$

in which we regard the $u$ 's and the $\bar{u}^{\prime}$ s as auxiliary variables. The sets of forms $\theta_{i}$ and $\bar{\theta}_{i}$ are equivalent in the above sense if and only if $\bar{x}^{i}, \bar{u}^{r}$ can be determined as functions of $x^{i}, u^{\bullet}(i, j=1, \cdots, n$; $r, s=1, \cdots, m)$ so that

$$
\bar{\omega}_{i}=\omega_{i} \quad i=1, \cdots, n .
$$

Such a system may be discussed by the general methods for dealing with Pfaffian systems. The first step is, of course, to adjoin to the system the equations $d \bar{\omega}_{i}=d \omega_{i}$; if we express the forms $d \omega_{i}$ by means of the forms $\omega_{i}$ themselves and of the differentials of the auxiliary variables, the coefficients of these expressions, when they contain the variables $x$ only, will yield invariants $I_{k}(x)$, and we may enlarge the original system by adjoining the equations $I_{k}(\bar{x})=I_{k}(x)$, together with the equations which are obtained from them by differentiation. 
Cartan shows that the continuation of this procedure eventually leads to a complete system of invariants which can be obtained by operations of differentiation only. However, when the systems one is led to consider are not in involution for the dimension $n$, the completeness of the system of invariants depends on the theorem that all singular solutions of a differential system may be obtained by the method of prolongation, a theorem which is not completely proved as yet (cf. above).

Among other applications which Cartan made of his theory of differential equations we mention the following: (1) Various applications to differential-geometric problems; (2) Principle of integral invariants in analytical dynamics; (3) Theory of general relativity.

As a matter of fact, the study of differential equations arising from problems of differential geometry had always interested him, and his papers on this subject run through most of his scientific career. The numerous examples given in [23] show quite decisively the advantages of using differential forms. One of the notable results is his proof of a conjecture of Schläfli to the effect that every Riemann metric of $n$ dimensions can be imbedded locally into a Euclidean space of dimension $n(n+1) / 2$ [22]. This theorem played no small part in Levi-Civita's original definition of his parallelism and has attracted the attention of differential geometers.

Also his work on integral invariants in analytical dynamics can be considered as an application of the theory of differential equations [21]. Mathematically the problem is to determine the trajectories, which are to be solutions of a differential system of the type

$$
\frac{d x_{i}}{d t}=X_{i}\left(x_{1}, \cdots, x_{n}, t\right), \quad i=1, \cdots, n .
$$

The standard way is by means of Hamilton's principle, which defines the trajectories as the extremals of a certain variational problem. Unfortunately the integrand of the latter does not have a simple physical interpretation. An alternative way was suggested by Poincaré. He called a multiple integral

$$
\int \cdots \int \sum_{i_{1}, \cdots, i_{p}} a_{i_{1}} \cdots i_{p}\left(x_{1}, \cdots, x_{n}, t\right) d x_{i_{1}} \cdots d x_{i_{p}}
$$

invariant when its value over a domain covered by the trajectories is invariant under the motion. In fact, it is called absolute if the domain is arbitrary and relative if the invariance is true only for closed domains. If $p_{i}, q_{i}, i=1, \cdots, n$, are the canonical variables of a dynamical system with $n$ degrees of freedom, Poincarés principle 
asserts that the trajectories can be characterized as the curves admitting the relative integral invariant

$$
\sum_{i=1}^{n} p_{i} d q_{i} \text {. }
$$

Cartan's principle is a modification of Poincaré's. He derived his ideas from his theory of differential systems. The differential system of the trajectories has $2 n-2$ functionally independent first integrals. Cartan observed that the property of an exterior differential form to be invariant and thus to depend only on the trajectories is that it is a form in these first integrals. Expressed in terms of the original variables, it may involve the independent variable $t$. Omitting from this the terms involving $d t$, we obtain the integrand of an invariant integral in the sense of Poincaré. Thus the latter is the truncated form of an invariant differential form (i.e., a form in the first integrals of the trajectories). Conversely, it can be proved that, given the integrand of an invariant integral of Poincaré, terms involving $d t$ can be added to it so as to obtain an invariant differential form. Cartan's principle characterizes the trajectories as admitting an invariant differential form. Moreover, the latter has a simple physical interpretation. The work therefore furnishes an interesting complement to formal dynamics.

In connection with the general theory of relativity and the unified field theory Cartan studied on several occasions the question of the possible forms of the equations of gravitation and of the unified gravitational and electro-magnetic field. He made a very detailed analysis and determined all possible forms of such differential systems. He was also the first one to introduce the notion of Riemann spaces without curvature and with torsion, which later served as the basis of Einstein's unified field theory. Apparently these studies are not of the same importance as his studies on pure mathematics.

\section{Geometry}

Although the theory of Lie groups has an intimate relationship with differential geometry, Cartan did not begin his substantial work on differential geometry until a relatively late stage. His first series of papers on differential geometry was concerned with the problem of deformation $[27 ; 28]$. It is clear that he had then all the essential ideas of the method of moving frames, one of his favorite subjects in later years, which has not been fully exploited even now.

The method was not new. It is a generalization, to an arbitrary homogeneous space, of the method of moving trihedrals, so success- 
fully used by Darboux, Ribaucour, and others [39; 41]. Even in the most general case some of its essential ideas had been given by Emile Cotton. It is also closely related to Cesaro's "intrinsic method" in differential geometry, as later developed by Kowalewski. To Cartan the attraction was not the method, but the geometrical results to which it so effectively leads. It is interesting to notice in his book [41] how he took pleasure in studying numerous examples and did not care to discuss the generalities, except in very sketchy outlines.

We attempt to give a description of this method in modern terminology. The problem is the local theory of a $p$-dimensional submanifold $M$ in a homogeneous space $E$ of dimension $n$, acted on by a Lie group $G$ of dimension $r$. Let $O$ be a point of $E$ and $H$ the subgroup of $G$ leaving $O$ fixed. Then the set of all transformations of $G$ carrying $O$ to a point $P$ of $E$ is a left coset $g H$ of $G$ relative to $H$, and $E$ can be identified with the space of left cosets $G / H$. Under this identification the action of $G$ on $E$ is represented by left multiplication. This process depends on the choice of $O$. If we replace $O$ by $O^{\prime}$ and if $g_{O}$ is a transformation of $G$ carrying $O^{\prime}$ to $O$, the subgroup of $G$ leaving $O^{\prime}$ fixed will be $g_{0}^{-1} H g_{0}$ and the set of transformations of $G$ carrying $O^{\prime}$ to $P$ will be $g H g_{o}$. In other words, the latter is defined up to multiplication by a fixed element to the right.

The method of moving frames is a method for the determination of differential invariants of $M$ under $G$, and in fact for determining enough of them to enable us to decide whether two given submanifolds differ from each other only by a transformation of $G$. Its main idea is that of passing from the homogeneous space $E$ to the group space $G$. In fact, denote by $\psi: G \rightarrow G / H$ the natural projection which assigns to an element $g \in G$ the coset $g H$. From $M$ we get the submanifold $F_{O}=\psi^{-1}(M) \subset G$, determined up to multiplication by a fixed element on the right (depending on the choice of the point $O$ ). $F_{O}$ is in general a manifold of dimension higher than $p$ and will be called the manifold of frames of order 0 of $M$. Now the Lie algebra $\mathfrak{h}$ of $H$ is a subalgebra of the Lie algebra $g$ of $G$. There is therefore in the dual space $\mathfrak{g}^{*}$ of $\mathfrak{g}$, whose elements are the so-called Maurer-Cartan forms, a linear subspace $\mathfrak{n}^{*}(G, H)$ of dimension $n$ consisting of all elements of $\mathfrak{g}^{*}$ orthogonal to $\mathfrak{h}$. The dual mapping of the identity mapping $i_{0}: F_{0} \rightarrow G$ maps the elements of $\mathfrak{n}^{*}(G, H)$ into Pfaffian forms on $F_{0}$, called by Cartan the principal components of order 0 . Among them there are exactly $p$ linearly independent ones, the others being their linear combinations. The coefficients of such linear combinations play an important rôle in the method of moving frames. From them 
it is sometimes possible to derive differential invariants of $M$ by elimination.

In order to get more information about $M$, we have to pass to the elements of contact of higher order. The general principle is to extend the above considerations to them. Let $M$ be defined locally by the equations

$$
x_{i}=f_{i}\left(u_{1}, \cdots, u_{p}\right), \quad i=1, \cdots, n,
$$

where the functions $f_{i}$ possess sufficiently many continuous partial derivatives or even are analytic. The values, at a certain point, of the functions $x_{i}$ and their partial derivatives up to the order $s$ inclusive, subject to the usual laws of transformation when either the $x$ 's or the $u$ 's undergo an admissible coordinate transformation, constitute an element of contact $C_{s}$ of order $s$. Thus an element of contact of order 0 is the point itself. Moreover, an element of contact of order $s>0$ determines uniquely an element of contact of order $s-1$, obtained by ignoring the derivatives of order $s$.

The totality of the elements of contact of order $s$, for all submanifolds of dimension $p$ in $E$, is a space $E_{z}$ on which $G$ acts. The elements of contact of order $s$ of $M$ constitute a submanifold $M_{\mathrm{s}}$ of $E_{8}$. The case for general $s$ differs from the case $s=0$ in two essential aspects: (1) The group $G$ does not necessarily act in a transitive manner on $E_{s}$, so that the latter decomposes into domains of transitivity; (2) The subgroup $H_{s}$ of $G$ leaving fixed a given $C_{s}$ may not be connected, as for instance in the case when $E$ is the Euclidean space with the group of rigid motions, and $p=1, s=1$. In this example $M$ is a curve and $C_{1}$ can be identified with the tangent direction; the motions leaving a line fixed have two connected components.

This phenomenon shows that the same $C_{s}$ may carry several "oriented" elements of contact of order $s$, obtained by replacing $H_{\text {s }}$ by the component of the unit element in $H_{s}$. The latter process involves an arbitrary choice, because only the class of conjugate subgroups of $H_{s}$ is defined by $C_{s}$. On the other hand, the first fact, together with generality assumptions, allows us to coordinatize the generic oriented elements of contact of order $s$ by a finite set of numbers. These are the differential invariants of order not greater than $s$.

Denote by $T$ a domain of transitivity of $E_{s}$ under $G$, by $H_{s}(T)$ the subgroup of $G$ leaving one of its points fixed, and by $H_{s}^{\prime}(T)$ the connected component of the unit element of $H_{b}(T)$. Then the space of oriented elements of contact of order $s$ can be identified with the union $\mathrm{U}_{T} G / H_{a}^{\prime}(T)$, and those of $M$ can be considered as a submani- 
fold $M_{s}$ of $\bigcup_{T} G / H_{s}^{\prime}(T)$. If $\psi_{s, T}: G \rightarrow G / H_{s}^{\prime}(T)$ is the natural projection, the submanifold

$$
F_{\mathrm{s}}=\bigcup_{C_{8} \in M_{0}} \psi_{\mathrm{s}, T}^{-1}\left(C_{8}\right)
$$

is called the manifold of frames of order $s$ of $M$. Generalizing the situation on frames of order 0 , let $\mathfrak{h}_{s}$ denote the Lie algebra of $H_{s}(T)$ and $\mathfrak{n}^{*}\left(G, H_{s}(T)\right)$ the linear subspace of the dual space $\mathfrak{g}^{*}$ of $\mathfrak{g}$ which consists of all elements of $\mathfrak{g}^{*}$ orthogonal to $\mathfrak{h}_{s}$. The dual mapping of the identity mapping $i_{s}: F_{s} \rightarrow G$ maps the elements of $\mathfrak{n}^{*}\left(G, H_{s}(T)\right)$ into Pfaffian forms on $F_{s}$, called the principal components of order not greater than $s$. The main feature of the method of moving frames is the result that the study of the manifold $F_{s}$ in $G$ gives some of the most important of the local geometric properties of $M$ in $E$. The determination of the manifolds of frames of different orders is achieved by induction on $s$.

Cartan had a more geometrical picture of the frames. To him they are configurations in $E$ such that there exists exactly one transformation of $G$ carrying one such configuration into another. In Euclidean space with the group of rigid motions we can take as frames sets of points $\left(P, U_{1}, U_{2}, U_{3}\right)$ with the properties: (1) the points $U_{1}, U_{2}, U_{3}$ are at a distance 1 from $P ;(2)$ any two of the lines $P U_{1}, P U_{2}, P U_{3}$ are perpendicular; (3) the vectors $P U_{1}, P U_{2}, P U_{3}$ form a righthanded system. For a surface in Euclidean space the frames of order 0 are those with $P$ on the surface. The frames of order one satisfy the further condition that $P U_{3}$ is normal to the surface. Here an orientation has to be made according as $U_{3}$ is along one sense of the normal or the other. If $P$ is not an umbilic, the frame of order two is uniquely determined at each point by the condition that $P U_{1}, P U_{2}$ are in the principal directions. The two principal curvatures are invariants of order two.

So far we have restricted our discussion to the generic elements of contact. Among the most interesting properties of differential geometry are perhaps those concerning the nongeneric ones. Thus the four-vertex theorem for closed plane curves and the theorem on the existence of umbilics on closed surfaces of genus $\neq 1$ are statements on the existence of certain types of elements of contact on a closed submanifold. No general result along this direction is known, and it is hoped that the method of moving frames will give some clues.

From this viewpoint the problem of deformation naturally presents itself. Two submanifolds $M^{p}$ and $M^{* p}$ in $E$ are said to be applicable or deformable of order $s$ relative to $G$ if there is a transformation of $G$ which carries the elements of contact of order $s$ of $M^{p}$ into those of 
$M^{* p}$, that is, if there is a one-one mapping between the submanifolds under which the invariants and the principal components of order not greater than $s$ are equal. When $G$ is the group of motions in Euclidean space and $p=2, s=1$, this notion of applicability reduces to the classical one studied by Gauss, Minding, Darboux, and others. When $G$ is the group of projective collineations in a real projective space and $p=2, s=2$, the problem was known as the projective deformation of surfaces and was studied at great length by Fubini and Cech.

When the order $s$ is sufficiently large, the method gives a solution of the fundamental problem of local differential geometry in a homogeneous space, namely, that of deciding whether two submanifolds differ from each other by a transformation of the group $G$.

Actually it may be quite complicated to carry out the method (i.e., to determine the invariants and frames of different orders) in concrete cases, particularly when $p$ is large. Moreover, the generality assumptions may soon become unrealistic. Cartan developed various ways of simplifying the computations and adapting the method to special cases. As frequently happens in mathematics, the generality of the viewpoint helps also to treat the special cases in a more effective way.

Some of the applications he made are: the conformal deformation of hypersurfaces [28], the projective deformation of surfaces [27], and the theory of submanifolds of constant curvature in a Euclidean or non-Euclidean space $[25 ; 26]$. The second was a problem which had received considerable attention from the Italian geometers. Cartan proved that, except for a class of surfaces which depends on six arbitrary functions of one variable, a surface is not deformable in a nontrivial way. Moreover, if a surface is projectively deformable (i.e., in a nontrivial way), the surfaces to which it is deformable depend at most on three arbitrary constants. This does not settle the problem. It perhaps makes the study of projectively deformable surfaces even more interesting. To cite an example, the question whether there exist surfaces projectively deformable to $\infty^{2}$ projectively inequivalent surfaces is not yet solved.

The study of the submanifolds of constant curvature in a Euclidean or non-Euclidean space generalizes the classical treatment of developable surfaces $[25 ; 26]$. Cartan made an exhaustive study and determined the degree of generality of such submanifolds. They do not exist under all circumstances. For instance, if the curvature of the submanifold of dimension $p$ is less than the curvature of the space, the latter must have dimension not less than $2 p-1$. His em- 
phasis was on the necessity of investigating the existence of submanifolds with prescribed properties, and their degree of generality, before their study can be undertaken with sense. For this purpose his theory of differential systems in involution was applied to the best advantage.

Most remarkable among his works along this line are the results on the isoparametric families of hypersurfaces in a spherical space $[42 ; 43]$. It started with a problem of Levi-Civita: to study the scalar functions in a Riemannian space which are functionally dependent on both their first and second Beltrami differential parameters. The hypersurfaces obtained by equating such a scalar function to a constant are said to form an isoparametric family. When the Riemannian space is of zero or constant negative curvature, the determination of its isoparametric families of hypersurfaces does not cause much difficulty. This is due to the fact that every such hypersurface has at most two distinct principal curvatures. When the Riemannian space is of constant positive curvature, the situation is very complicated, but also most interesting. Cartan proved that in this case there do exist isoparametric families of hypersurfaces having three distinct principal curvatures but that this can happen only when the dimension of the space is $4,7,13$, or 25 . The last family admits the exceptional simple Lie group in 52 parameters; this was the first time that this group was geometrically realized. Similar results hold for isoparametric families of hypersurfaces in spherical space with four distinct principal curvatures. These exist only in spaces of 5 and 9 dimensions. This is one of the few instances of geometrical problems where the dimension of the ambient space plays an essential rôle.

Einstein's theory of general relativity gave a new impetus to differential geometry. In their efforts to find an appropriate model of the universe geometers have broadened their horizon from the study of submanifolds in classical spaces (Euclidean, noneuclidean, projective, conformal, etc.) to that of more general spaces intrinsically defined. The result is an extension of the work of Gauss and Riemann on Riemannian geometry to spaces with a connection, which may be an affine connection, a Weyl connection, a projective connection, of a conformal connection. In these generalizations, sometimes called non-Riemannian geometry, an important tool is the absolute differential calculus of Ricci and Levi-Civita. The results achieved are of considerable geometric interest. For instance, in the theory of projective connections, developed independently by Cartan, Veblen, Eisenhart, and Thomas, it is shown that when the space has a system of 
paths defined by a system of differential equations of the second order, a generalized projective geometry can be defined in the space which reduces to ordinary projective geometry when the differential system is that of the straight lines. Numerous other examples can be cited. The problem at this stage is twofold: (1) to give a definition of "geometry" which will include most of the existing spaces of interest; (2) to develop analytic methods for the treatment of the new geometries, it being increasingly clear that the absolute differential calculus is inadequate.

For this purpose Cartan developed what seems to be the most comprehensive and satisfactory program and demonstrated its advantages in a decisive way $[31 ; 39]$. This contribution clearly illustrates his geometric insight and we consider it to be the most important among his works on differential geometry. It can be best explained by means of the modern notion of a fiber bundle. Let $p: B \rightarrow X$ be a fiber bundle with fiber $Y$ and structural group $G$. We assume $X$ to be a differentiable manifold and $G$ a Lie group. Let $U, V, W, \cdots$ be a covering of $X$ by coordinate neighborhoods. A point of $B$ belonging to $p^{-1}(U \cap V)$ has, with respect to $U$ and $V$, respectively, the coordinates $(x, y)$ and $\left(x, g_{U V}(x) y\right), x \in U \cap V, y \in Y$, where we denote the action of $G$ on $Y$ by multiplication to the left. The function $g_{U V}(x)$ is defined for $x \in U \cap V$, with values in $G$. Its dual mapping maps the Maurer-Cartan forms of $G$ into forms in $U \cap V$, to be denoted by $\omega_{U V}^{i}, i=1, \cdots, r$. Let $\left(a_{j}^{i}(g)\right), g \in G$, denote the adjoint representation of $G$ in the space of the Maurer-Cartan forms. A connection in the bundle is defined in each coordinate neighborhood $U$ by a set of Pfaffian forms $\theta_{U}^{\text {s }}, i=1, \cdots, r$, such that in $U \cap V$,

$$
\theta_{U}^{i}=\omega_{U V}^{i}+\sum_{j=1}^{r} a_{j}^{i}\left(g_{U V}\right) \theta_{v}^{j}, \quad i=1, \cdots, r .
$$

It is easy to verify that this condition is coherent in the intersection of any three coordinate neighborhoods $U, V, W$.

The curvature tensor is given by the exterior quadratic differential forms

$$
\Theta_{U}^{i}=d \theta_{U}^{i}-\frac{1}{2} \sum_{j, k=1}^{r} c_{c_{j k}^{i}}^{i} \theta_{U}^{j} \wedge \theta_{U}^{k}, \quad i=1, \cdots, r
$$

where the $c_{j k}$ are the constants of structure of $G$. Under a change of the coordinate neighborhood they are transformed according to the adjoint representation.

Actually Cartan proceeded in a different way. Guided by the clas- 
sical notion of parallelism he laid more emphasis on the possibility of "developing" the fiber along a parametrized curve. In the present formulation this possibility arises from the fact that the differential system

$$
\sum_{j=1}^{r} a_{j}^{i}(g) \theta^{j}+\omega^{i}(g)=0, \quad i=1, \cdots, r ; g \in G,
$$

is independent of the choice of the coordinate neighborhood. Corresponding to a parametrized curve in $X$ there is a uniquely determined integral curve $g(t)$ of the differential system through the unit element of $G$. The curve $g(t)$ gives rise to a one-parameter family of transformations in $Y$. Cartan called this process the development along a parametrized curve, and took it as the definition of a connection.

Without the notion and terminology of fiber bundles it was difficult to explain these concepts in a satisfactory way. The situation was further complicated by the fact that Cartan called tangent space what is now known as fiber while the base space $X$, being a differentiable manifold, has a tangent space from its differentiable structure. But he saw clearly that the geometrical situation demands the introduction of fiber bundles with rather general fibers. Attempts by several other mathematicians to tie up the fiber with the differentiable structure of the base space were suggested by their experience from affinely connected spaces and led to complicated computations which have nothing to do with the geometrical problem.

When one takes the notion of a connection as the guiding principle in differential geometry, the fundamental problem is to define the fiber bundle and the connection in every geometrical problem. This is not at all a routine matter, and Cartan carried it out in various cases. Some of the more important ones are: (1) the projective connection of the geometry of paths [29]; (2) the conformal connection in the conformal theory of Riemann spaces; (3) the metrical connection in Finsler spaces; (4) the metrical connection in spaces based on the notion of area of hypersurfaces, now known as Cartan spaces [38]; (5) the geometry of the integral $\int F\left(x, y, y^{\prime}, y^{\prime \prime}\right) d x$, which is a generalization of plane Finsler geometry.

Results from these particular examples tend to substantiate the belief that the notion of a connection is a guiding principle in differential geometry. For instance, in Finsler geometry, the spaces of interest are those defining a "regular" problem in the calculus of variations. This fact manifests itself clearly when one tries to define a connection in the space. 
In order that the connection in a space may be useful it must have a further property: it should give all the geometrical properties of the space. This can be made precise by the requirement that two spaces are to be equivalent under admissible transformations of the coordinates if and only if the connections are equivalent. This naturally leads to an equivalence problem whose analytical aspects have been discussed in Part II. It suffices to remark here that, while in simpler geometrical problems (such as Riemannian geometry) the introduction of a connection in the space automatically solves the equivalence problem, it is advisable in more general cases to go the other way around by first solving the equivalence problem and then interpreting the solution geometrically. Cartan's treatment of the equivalence problem furnishes a method which is particularly suitable for such geometrical problems.

Cartan, first in his Mémorial volume and later in his Leçons, applied his general ideas on connections to the case of Riemannian geometry $[30 ; 35]$. Although he himself never refrained from computations, he did not hide his distaste for the computational work on differential geometry which was then very fashionable and much of which was of little geometrical interest. He stated his aim in the preface of [35] as that of bringing out the simple geometrical facts which have often been hidden under a debauch of indices. The result is a very original account of Riemannian geometry, still the standard book in the field.

His most important work on Riemannian geometry is undoubtedly the theory of symmetric Riemann spaces $[33 ; 34 ; 37]$. It is well known that the local properties of a Riemann metric are given by Riemann-Christoffel curvature tensor and its successive covariant derivatives. Besides the locally Euclidean spaces the simplest Riemann spaces are therefore the ones for which the covariant derivative of the Riemann-Christoffel tensor is zero. These spaces, which include the Riemann spaces of constant curvature, are called symmetric by Cartan, for an obvious reason which will be brought out below. He published papers on the subject during the period from 1927 to 1935 . Perhaps because of their extensiveness the subject did not receive the attention it deserves. Its significance for the determination of the real forms of complex simple Lie groups has been discussed above. We proceed to give a very brief survey of the geometrical aspects of the theory, together with its relations to classical geometries, the theory of analytical functions of several complex variables, number theory, and topology.

Cartan soon discovered that the definition can be put in a more geometrical form. A symmetric Riemann space can be defined either 
as one for which the Levi-Civita parallelism preserves the sectional Riemannian curvature or as one for which the symmetry about a point is an isometry. From the second equivalent definition it follows immediately that the space admits a transitive group of isometries and that the connected component of the subgroup of isometries leaving fixed a point of the space is compact. This result brings the symmetric Riemann spaces into relation with homogeneous spaces.

The enumeration of all the symmetric Riemann spaces is not a simple problem. Cartan first observed that if a symmetric Riemann metric can be decomposed (locally) into a sum of two lower-dimensional Riemann metrics, each of the latter is symmetric. The problem is thus reduced to the irreducible case in which such a decomposition is not possible. Cartan then applied two different methods to the problem.

The first method consists in the determination of the subgroups of the orthogonal groups which can be the groups of holonomy of an irreducible symmetric Riemann space. Such a subgroup leaves invariant a form

$$
\sum R_{i j, k l} x^{i} y^{j} x^{b} y^{l}
$$

where $R_{i j k l}$ is the Riemann-Christoffel curvature tensor. It is thus not a most general subgroup of the orthogonal group, and this limitation makes it possible to carry out the program to the end. Unfortunately the method leads to very complicated computations.

It is the second method that opens up entirely unexpected views. Denote by $G$ the connected component of the group of all isometries, and by $H$ the connected component of the subgroup of $G$ leaving a point $O$ fixed. Then $H$ is compact. If $\sigma$ denotes the symmetry about $O$, the mapping which sends $g \in G$ into $\sigma g \sigma \in G$ is an involutory automorphism of $G$. Under this automorphism all elements of $H$ remain fixed. Conversely, when a connected Lie group $G$ has an involutory automorphism such that the connected component of the set of fixed elements is compact, the homogeneous space $G / H$ can be given a symmetric Riemann metric. Now choose a base in the Lie algebra of $G$ such that the endomorphism induced by the involutory automorphism changes the signs of some of the base vectors and leaves the remaining ones fixed. This normalization allows one to draw far-reaching conclusions on the infinitesimal structure of $G$. In fact, it follows that if the space, which we can now denote by $G / H$, is irreducible and is not locally Euclidean (that is, its RiemannianChristoffel tensor is not 0 ), the group $G$ is simple or is the direct product of two isomorphic compact simple groups. 
In the latter case the elements of $G$ can be written as $(a, b), a, b \in H$, $H$ being a simple group. Then the involutory automorphism has to be $(a, b) \rightarrow(b, a)$, and the space can be identified with the space of $H$. In other words, this case reduces to the geometry of the space of a compact simple Lie group.

More interesting is the case in which $G$ is simple. If $G$ is a complex simple Lie group and $H$ its compact real form, the space $G / H$ is homeomorphic to a Euclidean space and is the only symmetric Riemann space with $G$ as its group of isometries. Cartan called it the fundamental Riemann space of $G$. When $G$ is a noncompact simple real Lie group, we consider its corresponding complex group $G$ and the fundamental Riemann space $E$ of $G$. The involutory automorphism in $G$, which maps every element into its complex conjugate, induces a symmetry in $E$ leaving invariant a totally geodesic manifold of $E$. The latter is homeomorphic to a Euclidean space and is the only symmetric Riemann space with the group $G$. The situation is more complicated when $G$ is a compact simple real Lie group, as then the symmetric Riemann space with $G$ as the group of isometries is not unique. Thus there is, from the point of view of the infinitesimal structure, one and only one symmetric Riemann space belonging to a given noncompact simple group $G$ : it is that of the homogeneous space $G / H$, where $H$ is a maximal compact subgroup of $G$ (which turns out to be uniquely determined up to an inner automorphism of $G$ ). For instance, the symmetric Riemann space belonging to the unimodular real linear group $G L_{n}(R)$ is the space of positive definite quadratic forms in $n$ variables; to the unimodular complex linear group $G L_{n}(C)$ there belongs similarly the space of positive definite Hermitian forms in $n$ variables. This undoubtedly accounts for the rôle played by those forms both in classical and in modern number theory.

The study of symmetric Riemann spaces also throws considerable light on the relations between Riemannian geometry and the classical geometries, and helps to unify and explain some of the phenomena in classical geometries. Cartan carried out this idea for the case of complex projective geometry in his book [36]. It is known, for instance, that there is a correspondence between the geometry on the complex projective line and non-Euclidean hyperbolic geometry in space. In the present terminology the hyperbolic space is the fundamental Riemann space of the group of projective collineations on the complex projective line.

Actually Cartan's interest in symmetric Riemann spaces was aroused by a related but different problem. It is the study of Rie- 
mann spaces which admit an absolute parallelism, whose autoparallel curves are geodesics. The guiding example is the Clifford parallelism in non-Euclidean elliptic space. The first result in this direction was achieved jointly by Cartan and J. A. Schouten. They found that the irreducible spaces with absolute parallelism are exactly the spaces of compact simple Lie groups with one exception, which is the 7-dimensional elliptic space. The existence of the latter is related to properties of Cayley numbers.

Another application of the theory of symmetric Riemann spaces is to functions of several complex variables. Henri Cartan studied the group of all pseudo-conformal transformations which leave invariant a bounded domain in a space of several complex variables and proved that it is a Lie group. Using this result, Cartan studied the domains which are homogeneous, that is, which admit a transitive group of pseudo-conformal transformations. He did not succeed in determining all such domains, because there are perhaps too many. However, he did determine all those which are also symmetric, that is, which have the further property that to every point $O$ of the domain there exists an involutory pseudo-conformal transformation of the domain onto itself which admits $O$ as an isolated invariant point. This is due to the fact that the group is then semi-simple. The irreducible bounded symmetric homogeneous domains form four large classes and two exceptional cases, of dimensions 16 and 27 respectively. These domains have recently been found to play an important rôle in Siegel's work on automorphic functions and analytic number theory, where the discontinuous subgroups of these groups are studied. No bounded homogeneous domain is known which is not symmetric.

The notion of a symmetric space can be extended to the case which Cartan called non-Riemannian. It is a homogeneous space $G / H$ such that there is an involutory automorphism $\sigma$ of $G$ with $H$ as the connected component of the subgroup of invariant elements of $\sigma$, where $H$ is not necessarily compact. His main contribution to general symmetric homogeneous spaces is concerned with their Betti numbers, of which an account was given above in connection with the Betti numbers of compact Lie groups [11]. The results given there are valid for any compact symmetric homogeneous space, so that the determination of the Betti numbers of such a space can be reduced to a purely algebraic problem. Non-Riemannian symmetric spaces have otherwise hardly been studied.

Besides the importance of integral invariants in studying the topological properties of a space as a consequence of de Rham's theorems, 
they play a rôle in another field of geometry, now known as integral geometry. There again Cartan's exterior differential forms can be applied to the best advantage. In 1898 he devoted a paper to multiple integrals in the spaces of lines and planes of Euclidean space, which are invariant under the group of motions. The paper marks an important step in integral geometry, a subject founded by the English mathematician Croftoon and later developed by Blaschke and his school. It is quite curious that, although Cartan laid much emphasis on the idea of defining a group as the set of transformations leaving invariant a set of linear differential forms and took this to be the starting point of his theory of infinite groups, he did not come back to invariant differential forms of higher degree, except in the paper discussed above. His exterior differential forms have now become an indispensable tool in integral geometry.

\section{BibLIOGRAPHY}

The following is a partial list of Cartan's mathematical papers. It is complete in the papers published after 1939. A complete list of his papers up to 1939 can be found in [2], while [1] contains a complete list up to 1931 .

\section{SoURCES}

1. Notice sur les travaux scientifiques de M. Elie Cartan, Paris, Gauthier-Villars, 1931.

2. Selecta; Jubilê scientifique de M. Elie Cartan, Paris, Gauthier-Villars, 1939.

\section{GROUP THEORY}

3. Sur la structure des groupes de transformations finis et continus, Thèse, Paris, 1894; 2d ed., Paris, Vuibert, 1933.

4. Les groupes bilinéaires et les systèmes de nombres complexes, Ann. Fac. Sci. Univ. Toulouse vol. 12B (1898) pp. 1-99.

5. Les groupes projectifs qui ne laissent invariante aucune multiplicite plane, Bull. Soc. Math. France vol. 41 (1913) pp. 53-96.

6. Les groupes réels simples finis et continus, Ann. École Norm. vol. 31 (1914) pp. 263-355.

7. Les groupes projectifs continus réels qui ne laissent invariante aucune multiplicité plane, J. Math. Pures Appl. (6) vol. 10 (1914) pp. 149-186.

8. La géometrie des groupes de transformations, J. Math. Pures Appl. (9) vol. 6 (1927) pp. 1-119.

9. La géométrie des groupes simples, Ann. di Mat. vol. 4 (1927) pp. 209-256.

10. Groupes simples clos et ouverts et géometrie riemannienne, J. Math. Pures Appl. (9) vol. 8 (1929) pp. 1-33.

11. Sur les invariants intégraux de certains espaces homogènes clos et les proprietes topologiques de ces espaces, Annales de la Société Polonaise de Mathématiques vol. 8 (1929) pp. 181-225.

12. La theorie des groupes finis et continus et l'analysis situs, Mémorial des Sciences Mathématiques vol. 42, 1930. 
13. Les représentations linéaires des groupes de Lie, J. Math. Pures Appl. (9) vol. 17 (1938) pp. 1-12.

14. Leģons sur la theorie des spineurs I, II, Actualités Scientifiques et Industrielles, nos. $643,701,1938$.

\section{Differential equations}

15. Sur l'intégration des systèmes d'équations aux différentielles totales, Ann. École Norm. vol. 18 (1901) pp. 241-311.

16. Sur la structure des groupes infinis de transformations, Ann. Ecole Norm. vol. 21 (1904) pp. 153-206.

17. Sur la structure des groupes infinis de transformations, Ann. Ecole Norm. vol. 22 (1905) pp. 219-308.

18. Les sous-groupes des groupes continus de transformations, Ann. Ecole Norm. vol. 25 (1908) pp. 57-194.

19. Les groupes de transformations continus, infinis, simples, Ann. Ecole Norm. vol. 26 (1909) pp. 93-161.

20. Sur les équations de la gravitation d'Einstein, J. Math. Pures Appl. (9) vol. 1 (1922) pp. 141-203.

21. Leçons sur les invariants intégraux, Paris, Hermann, 1922.

22. Sur la possibilité de plonger un espace riemannien donné dans un espace euclidien, Annales de la Société Polonaise de Mathématiques vol. 6 (1927) pp. 1-7.

23. Les systèmes differentiels extérieurs et leurs applications géometriques, Paris, Hermann, 1945.

\section{Geometry}

24. Le principe de dualité et certaines intégrales multiples de l'espace tangentiel et d l'espace réglé, Bull. Soc. Math. France vol. 24 (1896) pp. 140-176.

25. Sur les variêtés de courbure constante d'un espace euclidien ou non euclidien, Bull. Soc. Math. France vol. 47 (1919) pp. 125-160.

26. Sur les varietes de courbure constante d'un espace euclidien ou non euclidien, Bull. Soc. Math. France vol. 48 (1920) pp. 132-208.

27. Sur la déformation projective des surfaces, Ann. École Norm. vol. 37 (1920) pp. 259-356.

28. Sur le problème gênéral de la déformation, C.R. Congrès Strasbourg, 1920, pp. $397-406$.

29. Sur les variêtés à connexion projective, Bull. Soc. Math. France vol. 52 (1924) pp. 205-241.

30. La géométrie des espaces de Riemann, Mémorial des Sciences Mathématiques, vol. 9, 1925. $1-42$.

31. Les groupes d'holonomie des espaces gênéralisés, Acta Math. vol. 48 (1926) pp.

32. (with J. A. Schouten), On the Riemannian geometries admitting an absolute parallelism, Neder. Akad. Wetensch. vol. 29 (1926) pp. 933-946.

33. Sur une classe remarquable d'espaces de Riemann, Bull. Soc. Math. France vol. 54 (1926) pp. 214-264.

34. Sur une classe remarquable d'espaces de Riemann, Bull. Soc. Math. France vol. 55 (1927) pp. 114-134.

35. Leçons sur la géométrie des espaces de Riemann, Paris, Gauthier-Villars, 1928; 2d ed., 1946.

36. Leçons sur la gémetrie projective complexe, Paris, Gauthier-Villars, 1931.

37. Les espaces riemanniens symétriques, Verh. Int. Math. Kong. Zurich, vol. I, 1932, pp. 152-161. 
38. Les espaces métriques fondés sur la notion d'aire, Actualités Scientifiques et Industrielles, no. 72, 1933.

39. La méthode du repère mobile, la théorie des groupes continus, et les espaces généralisés, Actualités Scientifiques et Industrielles no. 194, 1935.

40. Sur les domains bornés homogènes de l'espace de $n$ variables complexes, Abh. Math. Sem. Hamburgischen Univ. vol. 11 (1935) pp. 116-162.

41. La théorie des groupes finis et continus et la gémétrie différentielle, Paris, Gauthier-Villars, 1937.

42. Famille de surfaces isoparamétriques dans les espaces à courbure constante, Ann. di Mat. vol. 17 (1938) pp. 177-191.

43. Sur des familles remarquables d'hypersurfaces isoparametriques dans les espaces sphériques, Math. Zeit. vol. 45 (1939) pp. 335-367.

\section{PAPERS PUBLISHed AFTer 1939}

44. Sur quelques familles remarquables d'hypersurfaces, C.R. Congrès Sci. Math. Liège, 1939, pp. 30-41.

45. Sur les groupes linéaires quaternioniens, Vierteljahrsschrift der Naturforschenden Gesellschaft in Zurich vol. 85 (1940) pp. 191-203.

46. Sur des familles d'hypersurfaces isoparamétriques des espaces sphériques a $\mathbf{5}$ et a 9 dimensions, Universidad Nacional de Tucumán, Revista vol. A 1 (1940) pp. 5-22.

47. Sur un théorème de J. A. Schouten et W. van der Kulk, C.R. Acad. Sci. Paris vol. 211 (1940) pp. 21-24.

48. Sur une classe de surfaces apparentées aux surfaces $R$ et aux surfaces de Jonas, Mem. Vol. Dedicated to D. A. Grave, Moscow, 1940, pp. 72-78.

49. La geometria de las ecuaciones diferenciales de tercer orden, Revista Matemática Hispano-Americana vol. 1 (1941) pp. 1-31.

50. Sur les surfaces admettant une seconde forme fondamentale donnée, C.R. Acad. Sci. Paris vol. 212 (1941) pp. 825-828.

51. La notion d'orientation dans les différentes géométries, Bull. Soc. Math. France vol. 69 (1941) pp. 47-70.

52. Sur les couples de surfaces applicables avec conservation des courbures principales, Bull. Sci. Math. vol. 66 (1942) pp. 55-72, 74-85.

53. Notice sur M. Tullio Levi-Civita, C.R. Acad. Sci. Paris vol. 215 (1942) pp. 233-235.

54. Les surfaces qui admettent une seconde forme fondamentale donné, Bull. Sci. Math. vol. 67 (1943) pp. 8-32.

55. Sur une classe d'espaces de Weyl, Ann. École Norm. vol. 60 (1943) pp. 1-16.

56. Sur une classe de surfaces apparentées aux surfaces $R$ et aux surfaces de Jonas, Bull. Sci. Math. vol. 68 (1944) pp. 41-50.

57. Sur un probleme de gémétrie différentielle projective, Ann. École Norm. vol. 62 (1945) pp. 205-231.

58. Quelques remarques sur les 28 bitangentes d'une quartique plane et des 27 droites d'une surface cubique, Bull. Sci. Math. vol. 70 (1946) pp. 42-45.

59. L'oeuvre scientifique de M. E. Vessiot, Bull. Soc. Math. France vol. 75 (1947) pp. 1-8.

60. Sur l'espace annalagmatique réel à $n$ dimensions, Annales de la Société Polonaise de Mathématiques vol. 20 (1948) pp. 266-278. 